\title{
Período de incubación e intervalo serial para COVID-19 en una cadena de transmisión en Bahia Blanca (Argentina)
}

\author{
Incubation period and serial interval of Covid-19 \\ in a chain of infections in Bahia Blanca (Argentina)
}

Valentina Viego (https://orcid.org/0000-0001-5700-1133) ${ }^{1}$

Milva Geri (https://orcid.org/0000-0003-3265-3308) ${ }^{1}$

Juan Castiglia (https://orcid.org/0000-0002-3972-2946) ${ }^{2}$

Ezequiel Jouglard (https://orcid.org/0000-0002-5284-5422) ${ }^{3}$

${ }^{1}$ Departamento de Economía, Universidad Nacional del Sur. Av. Leandro Niceforo Alem 1253, Bahía Blanca. Buenos Aires Argentina. vviego@criba.edu.ar ${ }^{2}$ Departamento de Epidemiologia, Secretaria de Saúde do Município da Bahía Blanca. Buenos Aires Argentina.

${ }^{3}$ Departamento de Ciencias de la Salud, Universidad Nacional del Sur. Buenos Aires Argentina.

\begin{abstract}
The objective of this work was to estimate the incubation period and the serial interval of Covid-19 from a sample of symptomatic patients in Bahia Blanca city during the period March-May 2020. We collected dates of illness onset for primary cases and secondary cases for the first 18 secondary patients infected with SARSCov-2. Estimations of incubation period are based on a log-normal distribution while we assume a Gamma distribution for the serial interval. In both cases maximum likelihood estimator was applied to estimate main parameters. Of the total of 18 cases of local transmission analyzed, $17 \%$ occurred in the presymptomatic and asymptomatic phase. The mean incubation period for symptomatic patients is 7.9 days (95\% CI: 4.6, 11.1) considering the full sample and 7.5 days (95\%CI: 4.1, 10.9) if the sample is restricted to the most certain cases. The median is 6.1 and 5.8 days respectively. The point estimation for the mean serial interval is 6.8 days (95\% CI: 4.0-9.6). or 5.5 days (95\% CI: $2.8,8.1)$ for most certain pairs. The estimated median serial intervals were 5.2 and 4.1 days respectively. Comparisons with foreign estimates show that incubation period and serial interval could be longer in Bahia Blanca city than in other regions. Transmission from pre-symptomatic and asymptomatic is not negligible.
\end{abstract}

Key words Covid-19, Serial interval, Epidemiology, Bahia Blanca
Resumen El objetivo de este trabajo es estimar el periodo de incubación y el intervalo serial de Covid-19 en una muestra de pacientes sintomáticos en la ciudad de Bahía Blanca durante marzo-mayo de 2020. Se registraron fechas de aparición de síntomas de casos primarios y secundarios $y$ para los primeros 18 casos de transmisión local de SARS-Cov2 en Bahía Blanca (Argentina). La duración de la aparición de sintomas fue estimada suponiendo una distribución log normal. Para el intervalo serial se supuso una distribución gamma. Los parámetros fueron estimados por máxima verosimilitud. Del total de 18 casos de transmisión local analizados, $17 \%$ ocurrió en fase presintomática y asintomática. El período de incubación promedio es de 7.9 días (95\% IC 4.6, 11.1) y de 7.5 días $(95 \%$ IC 4.1, 10.9) si se toman los casos con mayor certidumbre sobre infectores $y$ fechas de exposición. La mediana es 6.1 y 5.8 días respectivamente. La estimación puntual del intervalo serial es 6.8 días (95\% IC: 4.0-9.6) y 5.5 días (95\% IC: $2.8,8.1$ ) considerando solo los pares más fiables. La mediana del intervalo serial se ubica en 5.2 y 4.1 dias respectivamente. La incubación y el intervalo serial podrían ser algo más largos en Bahía Blanca que en otras ciudades. La transmisión de asintomáticos y de pre-sintomáticos no es despreciable.

Palabras clave Covid-19, Intervalo serial, Epidemiología, Bahía Blanca 


\section{Introducción}

En Argentina el primer caso confirmado de la epidemia de Covid-19 fue reportado el 3 de marzo de 2020. A partir de la clasificación de enfermedad como pandemia por parte de la OMS, las autoridades sanitarias nacionales decidieron el aislamiento obligatorio de prácticamente toda la población desde el 20 de marzo para prevenir una rápida propagación del virus y preparar la expansión del sistema sanitario. A partir del 6 de abril, el gobierno fue relajando en forma secuencial las restricciones a las actividades comerciales y a los desplazamientos de población combinado con medidas de distanciamiento social, como el uso de barbijos y la preservación de distancia entre las personas.

Si bien se sabe que el aislamiento y las intervenciones que restringen los desplazamientos y contactos de la población detienen la propagación, aún hay datos insuficientes sobre cómo se transmite el virus dentro de las fronteras nacionales y en territorios específicos (pueblos, ciudades $\mathrm{o}$ áreas densamente pobladas). Además, como el testeo es limitado, no se conoce la proporción de asintomáticos o si el contagio tiene lugar durante la fase pre-sintomática. Más allá de las cifras básicas sobre casos confirmados, recuperados o fallecidos, el desarrollo de indicadores epidemiológicos es aún débil en regiones subdesarrolladas. En enfermedades infecciosas, uno de los indicadores clave es el intervalo serial (IS de aquí en adelante), definido como la fecha de aparición de síntomas de un infectado (caso secundario) y la fecha de síntomas de su infector (caso primario o índice). Este indicador contribuye a la comprensión de la transmisión de la enfermedad ${ }^{1}$. De hecho, el IS constituye uno de los insumos centrales en el cómputo del número reproductivo efectivo, es decir de la cantidad de infectados que cada transmisor genera ${ }^{2}$. Las estimaciones de IS sólo pueden obtenerse vinculando la fecha de síntomas entre pares de infectados-infectores. Por ello, la principal fuente de información surge de infecciones por conglomerado.

Actualmente, las cifras disponibles de IS para Covid-19 provienen de un puñado de regiones, en su mayoría de países con brote temprano ( $\mathrm{Ta}$ bla 1). Se ha señalado ${ }^{3}$ la necesidad de reunir más evidencia sobre el IS para evaluar intervenciones (rastreo de contactos, cuarentenas, etc.). Si el IS es sobreestimado las cuarentenas podrían ser excesivas. Por el contrario, si el IS se subestima, las intervenciones podrían ser insuficientes para detener la propagación. Al mismo tiempo, si el
IS es corto, los casos sintomáticos se manifiestan rápido y las autoridades sanitarias deben concentrar sus esfuerzos en la infraestructura de testeo y coordinación entre áreas de salud. Si el IS es largo, podría ser un signo de baja transmisión asintomática y representaría menor presión sobre los insumos de detección, al tiempo que permite realizar rastreos con menor premura.

Considerando el período de incubación, si la transmisión ocurre principalmente intra-hogar la fecha de exposición es incierta y restringe las estimaciones. Sólo se identificaron 6 estudios que ofrecen cifras del tiempo transcurrido entre la exposición y la aparición de síntomas (Tabla 2).

\section{Materiales y métodos}

Entre el 20 de marzo y el 8 de mayo de 2020 en Bahía Blanca (Argentina) fueron reportados 36 casos positivos para SARS-Cov2. Las autoridades sanitarias registraron la fecha de síntomas y la fecha de exposición de cada paciente. En tanto la mayoría de los contagios ocurrió en conglomerados fue posible identificar a los pares infectados-infector en la cadena.

De los casos totales, 13 de ellos fueron importados y 23 fueron contagios locales. Además, 2 de los 23 casos locales (17\%) se encontraban asintomáticos hasta el 8 de mayo.

Se cuenta con 17 observaciones de intervalos seriales individuales que surgen de 9 clusters de pacientes y 15 observaciones individuales sobre el período de incubación en pacientes que reportaron la fecha de exposición a su infector. En 3 de los casos, la fecha de exposición es imprecisa en tanto el infector identificado es probable. Por ello, la estimación del tiempo medio de incubación se hizo con la muestra completa y la muestra reducida a los casos con más certidumbre.

En la mayor parte de los pares (70\%) el infectado es parte del personal sanitario y contrajo el virus en el lugar de trabajo, en contacto estrecho con pacientes infectados. Para algunos de ellos (4 casos) el infector no pudo ser identificado con certidumbre, ya que más de un paciente pudo haber transmitido el virus. En esos casos, se asignó como infector a aquel que mostraba el menor lapso con los síntomas del infectado. Por ello, la muestra se dividió entre pares ciertos y pares probables $^{10}$. La submuestra de pares ciertos posee 17 observaciones.

Se considera que el IS sigue una distribución Gamma y que el tiempo de incubación sigue una distribución Log normal ${ }^{15}$. Los parámetros se es- 
Tabla 1. Estimaciones de intervalo serial de Covid-19. Evidencia internacional.

\begin{tabular}{|c|c|c|c|c|}
\hline Estudio & Region & Observaciones & Mediana & Media \\
\hline (4) & Wuhan & 6 & no reportado & $\begin{array}{c}7.5 \\
{[5.3-19.0]}\end{array}$ \\
\hline (5) & Corea del Sur & 12 & 4.0 & $\begin{array}{c}6.6 \\
{[3.0-9.0]}\end{array}$ \\
\hline (6) & China excl Hubei & 468 & no reportado & $\begin{array}{c}3.96 \\
{[3.53-4.39]}\end{array}$ \\
\hline (7) & China excl Hubei & 35 & no reportado & $\begin{array}{c}5.1 \\
{[1.3-11.6]}\end{array}$ \\
\hline (8) & China excl Hubei & 71 & 4.0 & $\begin{array}{c}4.27 \\
(3.44)\end{array}$ \\
\hline$(9)^{U}$ & Hong Kong & 21 & no reportado & $\begin{array}{c}4.4 \\
{[2.9-6.7]}\end{array}$ \\
\hline (10) & $\begin{array}{l}\text { Principalmente regiones } \\
\text { asiáticas y algunos casos en Alemania }\end{array}$ & $28^{\star}$ & 4.0 & $\begin{array}{c}4.7 \\
{[3.7-6.0]}\end{array}$ \\
\hline$(11)^{U}$ & Lombardia (Italia) & 90 & no reportado & $\begin{array}{c}6.6 \\
{[0.7-19]}\end{array}$ \\
\hline
\end{tabular}

Desvíos estandar entre paréntesis; IC al 95\% entre corchetes; ${ }^{\star}$ Los autores también proveen estimaciones para 18 pares con certidumbre sobre fechas de síntomas; ${ }^{\mathrm{U}}=$ No publicado, en proceso de evaluación por pares Fuente: propia basada en referencias.

Tabla 2. Período de incubación de Covid-19. Evidencia internacional.

\begin{tabular}{|c|c|c|c|c|}
\hline Estudio & Region & $\mathbf{N}$ & Media -dias- & Desvío est -días- \\
\hline (12) & viajeros desde y hacia Wuhan (China) & 88 & $\begin{array}{c}6.4 \\
{[5.6-7.7]}\end{array}$ & $\begin{array}{c}2.3 \\
{[1.7-3.7]}\end{array}$ \\
\hline (4) & China & 10 & $\begin{array}{c}5.2 \\
{[4.1-7.0]}\end{array}$ & no report \\
\hline (13) & China excl Hubei & 181 & $\begin{array}{c}5.1 \mathrm{M} \\
{[4.5-5.8]}\end{array}$ & no report \\
\hline (14) & China excl Wuhan & 52 & $\begin{array}{c}5.0 \\
{[4.2-6.0]^{*}}\end{array}$ & $3[2.1-4.5]$ \\
\hline (7) & China excl Hubei & 49 & $\begin{array}{c}5.2 \\
{[1.8-12.4]}\end{array}$ & no report \\
\hline (8) & China excl Hubei & 65 & $\begin{array}{c}5.33 \\
5.0 \mathrm{M}\end{array}$ & 3.3 \\
\hline
\end{tabular}

$\mathrm{M}=$ mediana; ${ }^{*}$ La media es alrededor de 5.6 con desvío 2.8 si se incluyen habitantes de Wuhan.

Fuente: Propia basada en referencias.

timaron con el comando fitdist del paquete fitdistplus de R aplicando el estimador de máxima verosimilitud, aceptado como el mejor método para obtener estimaciones de tiempo hasta el evento en datos de pacientes ${ }^{16,17}$. Los intervalos de confianza para la mediana se obtuvieron usando el estimador de pareo por cuartiles (quantile matching).

Además se estimó la media del IS de la muestra de pacientes suponiendo incertidumbre en las fechas probables de síntomas. Esto requiere la aplicación de un enfoque bayesiano, basado en la técnica numérica conocida como cadenas de Markov de Monte Carlo ( $m c m c$ por sus siglas en inglés). Este procedimiento estima una distribución muestral posterior para el IS, que es una especie de promedio entre las creencias previas sobre la distribución de la variable (e.g. Gamma) y los datos observados. La estimación bayesiana puede ser más acertada en fenómenos con menor conocimiento de su dinámica, como la Covid-19. 


\section{Resultados}

La Figura 1 representa la cadena de contagios ( 2 casos importados y 18 casos de transmisión local). El resto de los 13 casos importados no generaron contagio local. A la fecha de corte, todos los casos se encontraban recuperados. Por este motivo, la cadena de contagios de los casos analizados no padece censura a la derecha.

Se detectaron 2 casos de transmisión pre-sintomática, en tanto el infector manifestó síntomas luego del contacto con su infectado. Además, se detectó un caso de transmisión de asintomático. Esto indica que $17 \%$ de los contagios ocurrió en fase asintomática o pre-sintomática.

Como el número de observaciones es modesto y los métodos paramétricos tienen propiedades asintóticas, también se chequearon los resultados con técnicas de re-muestreo (Cuadro 1).

Los resultados se presentan en la Tabla 3. El período de incubación promedio para pacientes sintomáticos es de 7.9 días (95\% IC 4.6, 11.1) considerando la muestra de 15 pacientes y de 7.5 días (95\% IC 4.1, 10.9) si se toman los casos con mayor certidumbre sobre infectores y fechas de exposición $(\mathrm{n}=12)$. La mediana es 6.1 (95\% IC: $4.1,9.2)$ y $5.8(95 \%$ IC $3.6,9.3)$ días respectivamente.

De los datos locales surge que $97.5 \%$ de los sintomáticos presentará síntomas antes de los 13/14 días, pero si se considera la muestra reducida de 12 pacientes este evento podría ocurrir a los 17 días de la exposición. Este hallazgo tiene implicancias sobre el tiempo de aislamiento de casos sospechosos (expuestos a confirmados).

La estimación puntual del intervalo serial es 6.8 días (95\%IC: 4.0-9.6). Considerando solamente los pares más fiables, el intervalo serial promedio es de 5.5 días (95\%IC: 2.8, 8.1). Las medianas del intervalo serial fueron 5.2 (95\%IC: $3.0,8.1)$ y 4.1 (95\%IC: $2.0,6.9)$ días respectivamente.

No se encontraron diferencias sustanciales en las estimaciones puntuales entre métodos paramétricos tradicionales y los bayesianos, aunque las estimaciones bayesianas tienen mayores

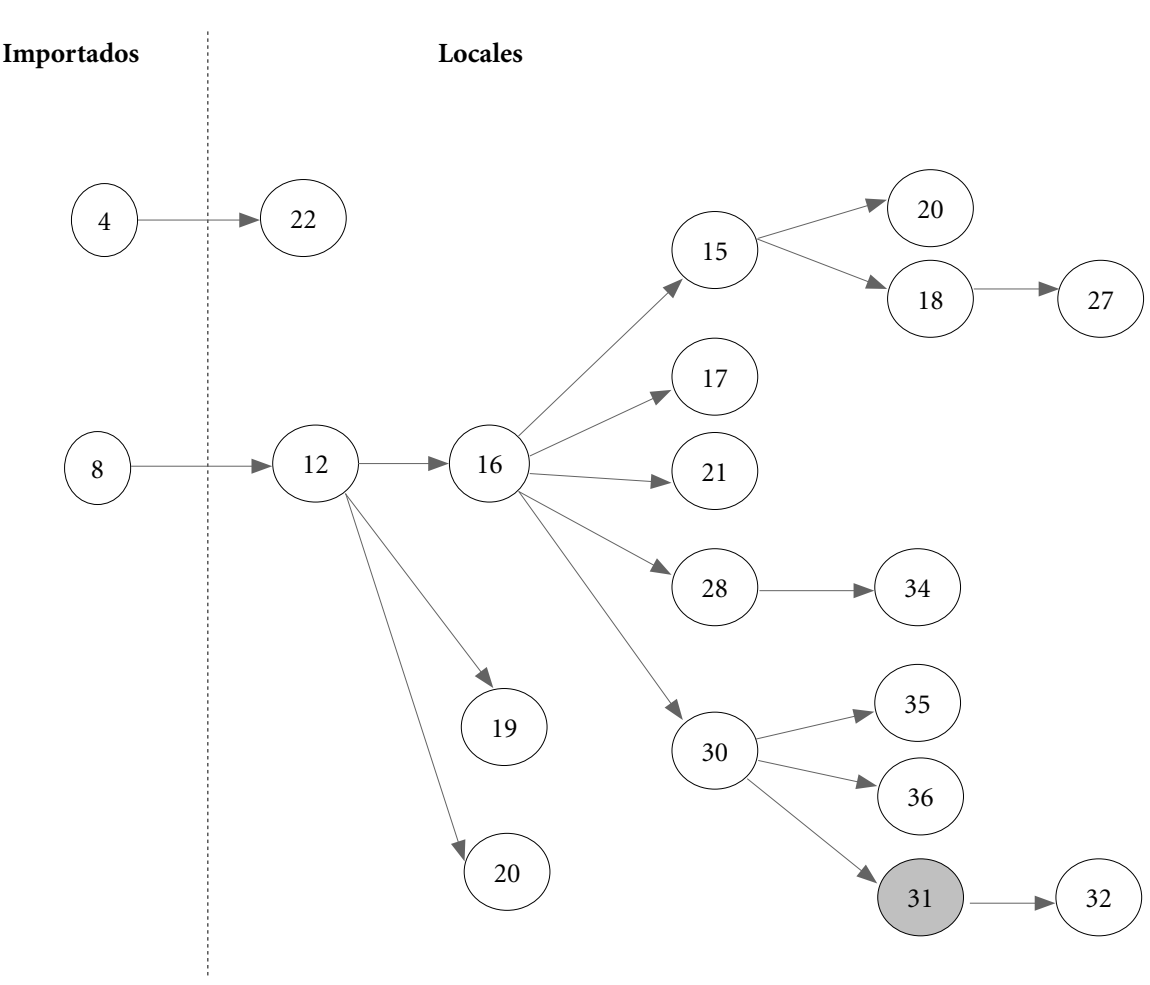

Figura 1. Covid-19. Cadena de contagios en una muestra de pacientes de Bahía Blanca. 20-marzo a 8-mayo, 2020. 
Tabla 3. Período de incubación e intervalo serial en una muestra de infectados sintomáticos.

\begin{tabular}{|c|c|c|c|c|c|c|}
\hline & $\begin{array}{c}\text { Período de } \\
\text { incubación } \\
\text { muestra } \\
\text { completa }\end{array}$ & $\begin{array}{l}\text { Período de } \\
\text { incubación } \\
\text { muestra } \\
\text { reducida }\end{array}$ & $\begin{array}{c}\text { Intervalo } \\
\text { serial } \\
\text { muestra } \\
\text { completa }\end{array}$ & $\begin{array}{c}\text { Intervalo } \\
\text { serial } \\
\text { muestra } \\
\text { reducida }\end{array}$ & $\begin{array}{c}\text { Intervalo } \\
\text { serial muestra } \\
\text { completa } \\
\text { enfoque } \\
\text { bayesiano } \\
\end{array}$ & $\begin{array}{c}\text { Intervalo } \\
\text { serial muestra } \\
\text { reducida } \\
\text { enfoque } \\
\text { bayesiano }\end{array}$ \\
\hline Distribución & Log normal & Log normal & Gamma & Gamma & Gamma $^{\star *}$ & Gamma ${ }^{* *}$ \\
\hline \multirow{2}{*}{ Media (1) } & 7.86 & 7.50 & 6.82 & 5.46 & 6.88 & 6.12 \\
\hline & [4.63-11.09] & [4.11-10.89] & [4.04-9.61] & [2.84-8.08] & {$[2.0-32.14]$} & [1.31-31.73] \\
\hline \multirow{2}{*}{$\begin{array}{l}\text { Desvío } \\
\text { estándar (2) }\end{array}$} & 6.38 & 6.23 & 5.86 & 4.81 & 5.89 & 4.55 \\
\hline & [3.42-15.87] & [3.21-16.99] & [1.80-3.69] & [2.48-13.12] & [2.09-17.76] & [1.47-16.77] \\
\hline \multirow{2}{*}{ Mediana ${ }^{\star}$} & 6.1 & 5.76 & 5.24 & 4.13 & 6.4 & 5.15 \\
\hline & {$\left[4.06-9.18^{\star}\right]$} & {$\left[3.56-9.33^{\star}\right]$} & {$[2.96-8.11]^{\star}$} & {$\left[2.04-6.93^{\star}\right]$} & [4.6-9.2] & {$[2.95-7.50]$} \\
\hline $\mathrm{CV}(2) /(1)$ & 0.81 & 0.83 & 0.86 & 0.88 & 0.86 & 0.74 \\
\hline n & 15 & 12 & 17 & 13 & 17 & 13 \\
\hline $\ln \mathrm{L}$ & -43.31 & -34.19 & -49.21 & -34.83 & -52.52 & -37.96 \\
\hline
\end{tabular}

intervalos creíbles, lo cual es esperable en tanto el método admite mayor incertidumbre en la estimación.

La Figura 2 presenta los histogramas y el ajuste de las distribuciones a los datos. Se observa un mejor ajuste para el IS que para el tiempo de incubación.

\section{Discusión}

La media del período de incubación de Covid-19 estimada para Bahía Blanca parece ser mayor que la informada para regiones asiáticas. Esto implica una propagación posiblemente más lenta en la medida en que no exista contagio asintomático ni pre-sintomático. De todos modos, se debe advertir que, como el coeficiente de variación es mayor en el caso local respecto del reportado para otras regiones, esto indica que la heterogeneidad de la propagación local es también mayor que la estimada para las regiones y/o que el tamaño muestral es todavía bajo para arrojar estimaciones más precisas. No obstante ${ }^{4}$, se basa en una muestra reducida de 10 casos y también presenta menor variación que el caso local, lo cual podría 

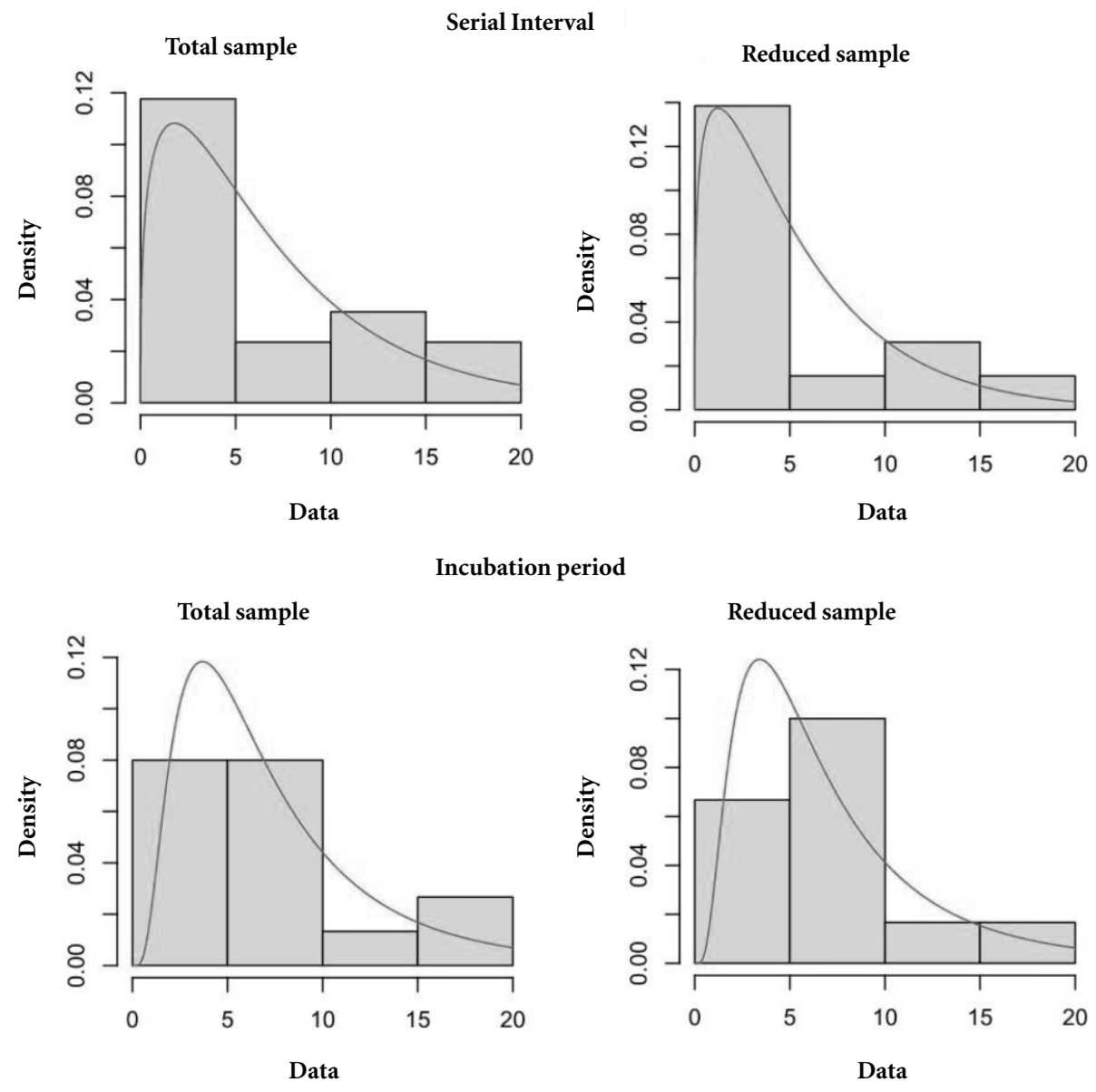

Figura 2. Densidad teórica y empírica del intervalo serial y del período de incubación para la muestra total y reducida. Resultados de la estimación paramétrica.

Fuente: elaboración propia.

favorecer la hipótesis de una mayor dispersión local respecto de la evidencia internacional.

Por otro lado, las estimaciones muestran que en algunos casos extremos el período de incubación podría demorar 17 días. Esto implica que medidas de aislamiento de casos sospechosos (contactos estrechos de casos positivos) de 2 semanas podrían ser insuficientes. El aislamiento de 2,5 semanas sería más precautorio.

Las estimaciones del IS de Covid-19 sugieren que la aparición de síntomas entre un infectado y el subsiguiente puede tomar más días que los observados en regiones asiáticas y es más cercano a las cifras reportadas para la región de Lombardía (Italia). Esto incrementa el valor del número reproductivo efectivo (para un crecimiento dado de los nuevos casos). Pero, al mismo tiempo, cifras de IS más bajas también implican menor aglomeración de casos en el sistema de salud y, por ende, menor presión sobre recursos del sistema.

La brecha entre el período de incubación y el IS es similar en magnitud que la estimada en otras regiones del mundo; confirmando que en Bahía Blanca la transmisión puede ocurrir entre 1 y 2 días previos a la aparición de síntomas. Esto destaca la importancia del rastreo de casos y las medidas de aislamiento efectivas de los contactos estrechos de los confirmados.

El estudio tiene algunas limitaciones. Primero, de los primeros 36 casos, un tercio de los sintomáticos corresponden a personal sanitario, en el cual el contagio ocurrió claramente durante la fase sin- 
tomática del infector y no antes. Por este motivo, el tiempo de contagio pre-sintomático podría ser incluso mayor. Para chequear esto es necesaria más evidencia de contagios en casos fuera del sistema de salud.

\section{Aprobación del comité de ética}

Los datos están anonimizados para proteger la identidad del paciente. El trabajo fue aprobado por unanimidad por los miembros del Comité Institucional de Etica del Hospital Municipal Leonidas Lucero, de Bahía Blanca.

\section{Colaboradores}

La recolección de los datos estuvo a cargo de J Castiglia y E Jouglard. V Viego y M Geri realizaron las estimaciones y el informe final.

\section{Financiamiento}

La investigación no recibió fondos especiales. $\mathrm{V}$ Viego y M Geri son parte del equipo de investigadores del Instituto de Investigaciones Económicas y Sociales del Sur (IIESS-Conicet). J Castiglia y E Jouglard forman parte del Departamento de Epidemiología del Hospital Municipal Leónidas Lucero. 


\section{Referencias}

1. Fine PEM. The interval between successive cases of an infectious disease. Am J Epidem 2003; 158(11):10391047.

2. Wallinga J, Lipstich M. How generation intervals shape the relationship between growth rates and reproductive numbers. Proc Biol Sci 2007; 274:599-604.

3. Peak C, Kahn R Grad Y, Childs L, Li R, Lipsicth M, Buckee CO. Individual quarantine versus active monitoring of contacts for the mitigation of Covid-19: a modelling study. Lancet Inf Dis 2020; S14733099(20)30361-3.

4. Li Q, Guan X, Wu P, Wang X, Zhou L, Tong Y, Ren R, Leung KSM, Lau EHY, Wong JY, Xing Xuesen, Xiang N, Wu Yang, Li C, Chen Q, Li D, Liu T, Zhao J, Liu M, Tu W, Chen C, Jin L, Yang R, Wang Q, Zhou S, Wang R, Liu H, Luo Y, Liu Y, Shao G, Li H, Tao Z, Yang Y, Deng Z, Liu B, Ma Z, Zhang Y, Shi G, Lam TTY, Wu JT, Gao GF, Cowling BJ, Yang B, Leung GM, Feng Z. Early transmission dynamics in Wuhan, China, of novel coronavirus-infected pneumonia. N Engl J Med 2020; 382:1199-1207.

5. Ki M. Epidemiologic Characteristics of Early Cases With 2019 Novel Coronavirus (2019-nCoV) Disease in Korea. Epidemiol Health 2020; 42:e2020007.

6. Du Z, Xu X, Wu Y, Wang L, Cowling B, Meyer A. Serial interval of Covid-19 among publicly reported confirmed cases. Emerging Inf Dis 2020; 26(6):1341-1343.

7. Zhang J, Litvinova M, Wang W, Wang Y, Deng X, Chen X, Li M, Zheng W, Yi L, Chen X, Wu Q, Liang Y, Wang $\mathrm{X}$, Yang J, Sun K, Longini Jr IM, Halloran E, Wu P, Cowling BJ, Merler S, Viboud C, Vespignani A, Ajelli M, Yu $\mathrm{H}$. Evolving epidemiology and transmission dynamics of coronavirus disease 2019 outside Hubei province, China: a descriptive and modelling study. Lancet Inf Dis 2020: 793-802.

8. Zhou X, You C, Deng Y, Hu W, Sun J, Lin Q, Zhou F, Pang CH, Zhang Y, Chen Z, Zhou X-H. Estimation of the Time-Varying Reproduction Number of COVID-19 Outbreak in China. Lancet Public Health 2020 [preprint]. [acessado 2020 Jun 05]. Disponível em: https://doi.org/10.1101/2020.02.08.20021253

9. Zhao S, Gao D, Zhuang Z, Chong M, Cai Y, Ran J, Cao P, Wang K, Lou Y, Wang W, Yang L, He D, Wang M. Estimating the serial interval of the novel coronavirus disease (COVID-19): A statistical analysis using the public data in Hong Kong from January 16 to February 15, 2020.MedRX 2020 [preprint].

10. Nishiura H, Linton N, Akhmetzhanov A. Serial interval of novel coronavirus (COVID 19) infections. Int J Inf Dis 2020; 93:284-286.
11. Cereda D, Tirani M, Rovida F, Demicheli V, Ajelli M, Poletti P, Trentini F, Guzzeta G, Marziano V, Barone A, Magoni M, Deandrea S, Diurno G, Lombardo M, Faccini M, Pan A, Bruno R, Pariani E, Grasselli G, Piatti A, Gramegna M, Baldanti F, Melegaro A, Merler S. The early phase of the COVID-19 outbreak in Lombardy, Italy. ArXiv 2020 [preprint].

12. Backer JA, Klinkenberg D, Wallinga J. Incubation period of 2019 novel coronavirus (2019nCoV) infections among travellers from Wuhan, China, 20-28 January 2020. Euro Surveill 2020; 25(5):pii $=2000062$.

13. Lauer S, Grantz K, Bi Q, Jones F, Zheng Q, Meredith H, Azman AS, Reich NG, Lessler J. The incubation period of coronavirus disease 2019 (Covid 19) from publicly reported confirmed cases: estimation and application. Ann Int Med 2020; 172(9):577-582.

14. Linton NM, Kobayashi T, Yang Y, Hayashi K, Akhmetzhanov AR, Jung S, Yuan B, Kinoshita R, Nishiura H. Incubation period and other epidemiological characteristics of 2019 novel coronavirus infections with right truncation: a statistical analysis of publicly available case data. J Clin Med 2020; 9(2):538.

15. Lessler J, Reich NG, Brookmeyer R, Perl TM, Nelson $\mathrm{KE}$, Cummings DAT. Incubation periods of acute respiratory viral infections: a systematic review. Lancet Infect Dis 2009; 9:291-300.

16. Wallinga J, Teunis P. Different epidemic curves for severe acute respiratory syndrome reveal similar impacts of control measures. Am J Epidemiol 2004; 160(6):509-516.

17. Bolker B. Ecological models and data in R. Princeton: Princeton Univ Press; 2008.

Artículo presentado en 17/06/2020

Aprobado en 18/06/2020

Versión final presentada en 20/06/2020 